\title{
The effect of immediate breast reconstruction on the timing of adjuvant chemotherapy: a systematic review
}

\author{
J. Xavier Harmeling ${ }^{1}$ Casimir A. E. Kouwenberg ${ }^{1} \cdot$ Eveline Bijlard $^{1}$ • \\ Koert N. J. Burger ${ }^{2} \cdot$ Agnes Jager $^{3} \cdot$ Marc A. M. Mureau $^{1}$
}

Received: 6 March 2015/Accepted: 10 August 2015/Published online: 19 August 2015

(c) The Author(s) 2015. This article is published with open access at Springerlink.com

\begin{abstract}
Adjuvant chemotherapy is often needed to achieve adequate breast cancer control. The increasing popularity of immediate breast reconstruction (IBR) raises concerns that this procedure may delay the time to adjuvant chemotherapy (TTC), which may negatively impact oncological outcome. The current systematic review aims to investigate this effect. During October 2014, a systematic search for clinical studies was performed in six databases with keywords related to breast reconstruction and chemotherapy. Eligible studies met the following inclusion criteria: (1) research population consisted of women receiving therapeutic mastectomy, (2) comparison of IBR with mastectomy only groups, (3) TTC was clearly presented and mentioned as outcome measure, and (4) original studies only (e.g., cohort study, randomized controlled trial, case-control). Fourteen studies were included, representing 5270 patients who had received adjuvant chemotherapy, of whom 1942 had undergone IBR and 3328 mastectomy only. One study found a significantly shorter mean TTC of 12.6 days after IBR, four studies
\end{abstract}

Electronic supplementary material The online version of this article (doi:10.1007/s10549-015-3539-4) contains supplementary material, which is available to authorized users.

Marc A. M. Mureau

m.mureau@erasmusmc.nl

1 Department of Plastic and Reconstructive Surgery, Erasmus MC Cancer Institute, University Medical Center Rotterdam, PO Box 2040, 3000 CA Rotterdam, The Netherlands

2 Kennisinstituut van Medisch Specialisten, PO Box 3320, 3502 GH Utrecht, The Netherlands

3 Department of Medical Oncology, Erasmus MC Cancer Institute, University Medical Center Rotterdam, PO Box 5201, 3008 AE Rotterdam, The Netherlands found a significant delay after IBR averaging 6.6-16.8 days, seven studies found no significant difference in TTC between IBR and mastectomy only, and two studies did not perform statistical analyses for comparison. In studies that measured TTC from surgery, mean TTC varied from 29 to 61 days for IBR and from 21 to 60 days for mastectomy only. This systematic review of the current literature showed that IBR does not necessarily delay the start of adjuvant chemotherapy to a clinically relevant extent, suggesting that in general IBR is a valid option for non-metastatic breast cancer patients.

Keywords Immediate breast reconstruction .

Chemotherapy · Mastectomy $\cdot$ Breast cancer $\cdot$ Systematic review

\section{Introduction}

One out of every eight women will be diagnosed with breast cancer in her lifetime, making it the most common cancer in women [1]. Over the last decades the survival rate has increased slowly, which is currently estimated to be $85 \%$ in developed countries [2]. However, with an estimated annual number of breast cancer deaths of 537,000 worldwide, breast cancer still is the most important cause of death by cancer among women [3].

Despite advances in different treatment modalities, about $45 \%$ of all breast cancer patients still undergoes a mastectomy for adequate local control $[4,5]$. The resulting loss of a breast may have a negative effect on body image, sexuality, and sense of femininity [6]. Breast reconstruction aims to diminish the negative psychological impact of mastectomy and to improve patients' quality of life. Currently, approximately $20-40 \%$ of women who undergo a 
mastectomy receive breast reconstruction [1]. Breast reconstruction can either be performed immediately following mastectomy during the same operation or as a delayed procedure after completion of the entire oncologic treatment. Immediate breast reconstruction (IBR) has several reported advantages, such as favorable esthetic outcomes and less psychological burden, avoiding additional operations, hospitalizations, and costs [4].

A disadvantage of IBR is the increased risk of postoperative complications, which causes concerns regarding oncological safety [7]. Almost $39 \%$ of medical oncologists and $23 \%$ of surgical oncologists feel that breast reconstruction adversely interferes with adjuvant oncological therapy [8]. One concern is that IBR increases the time to adjuvant chemotherapy (TTC), which may have a negative impact on recurrence and survival rates. To put a possible delay in the administration of adjuvant chemotherapy into perspective, it needs to be established from which point on this delay negatively affects oncological safety and, hence, becomes clinically relevant.

Various studies aimed at identifying the cut-off point after which increased TTC has a significant negative impact on survival. In relation to relapse-free survival, disease-free survival or overall survival in non-metastatic breast cancer patients, no such cut-off point has been identified, given chemotherapy was started within 3 months after surgery [9-13]. One study, however, identified a subgroup of premenopausal patients with ER-negative node-positive tumors who showed impaired diseasefree survival if chemotherapy was started 21-86 days versus within 20 days after surgery [10]. Furthermore, in patients with stage I or II breast cancer relapse-free survival and overall survival were found to significantly decrease if chemotherapy was postponed more than 3 months after surgery [12].

The purpose of this study was to perform a systematic review of the current literature to investigate whether TTC is affected by IBR compared with mastectomy only.

\section{Methods}

Within the databases Embase, Medline (PubMed), Web of Science, Scopus, the Cochrane Central, and Google Scholar studies on the effect of IBR on TTC in breast cancer patients were searched on 6 October 2014. Keywords related to breast reconstruction, chemotherapy, and a clinical study design were used. The exact search string is shown in the appendix. No limitations were placed on study design, language, or otherwise. References were checked for duplicity and deleted accordingly.

Two reviewers (JXH and CAEK) independently assessed the title and abstract of all references with the following inclusion criteria: (1) the research population consists of women undergoing a mastectomy for breast cancer, (2) the study compares a cohort receiving IBR with one receiving mastectomy only, (3) timing of adjuvant chemotherapy is mentioned as outcome measure and is appropriately quantified, and (4) the publication concerns an original study (i.e., cohort study, randomized controlled trial, case-control, case study). Conference abstracts and reviews were excluded. In case of disagreement between two reviewers, a third reviewer (EB) made the final decision.

Subsequently, the full text of the selected studies was reviewed for final inclusion. If deemed necessary, authors were contacted with a request to provide additional information or clarification. Next, the reference lists of these finally included studies were searched for references to other relevant studies, which had not been included in the original search. The selection of these references was performed using the same criteria as mentioned above.

Study quality was assessed through an estimation of bias due to various causes [14]. Data were extracted using a predefined extraction form. Information was obtained on study design, patient characteristics (including comorbidities), outcome measures regarding TTC, and variables regarding other aspects of adjuvant chemotherapy.

\section{Statistical analysis}

If only median values were reported, authors were contacted with a request to provide mean values and standard deviations, to enable us to calculate $95 \%$ confidence intervals of the mean differences.

Clinical and statistical heterogeneity were assessed and, if deemed sufficiently low, a meta-analysis was performed using pooled data. Statistical heterogeneity was determined using the $\mathrm{I}^{2}$ index. An $\mathrm{I}^{2}$ value smaller than $25 \%$ was considered to indicate low heterogeneity, a value of $25-50 \%$ moderate heterogeneity, and a value above $50 \%$ high heterogeneity [15]. If $\mathrm{I}^{2}$ was low or moderate a fixed effects model was used, whereas we used a random effects model if the $\mathrm{I}^{2}$ index indicated high heterogeneity. Overall effect was calculated as a $Z$-statistic, with $95 \%$ confidence intervals, and regarding a $p$ value less than 0.05 as significant. Review Manager 5.3 (Copenhagen: The Nordic Cochrane Centre, The Cochrane Collaboration, 2014) was used for statistical analysis.

\section{Results}

The literature search yielded 1978 unique publications and after applying the selection criteria 25 publications were read in full text, of which 14 were finally included (Fig. 1) 


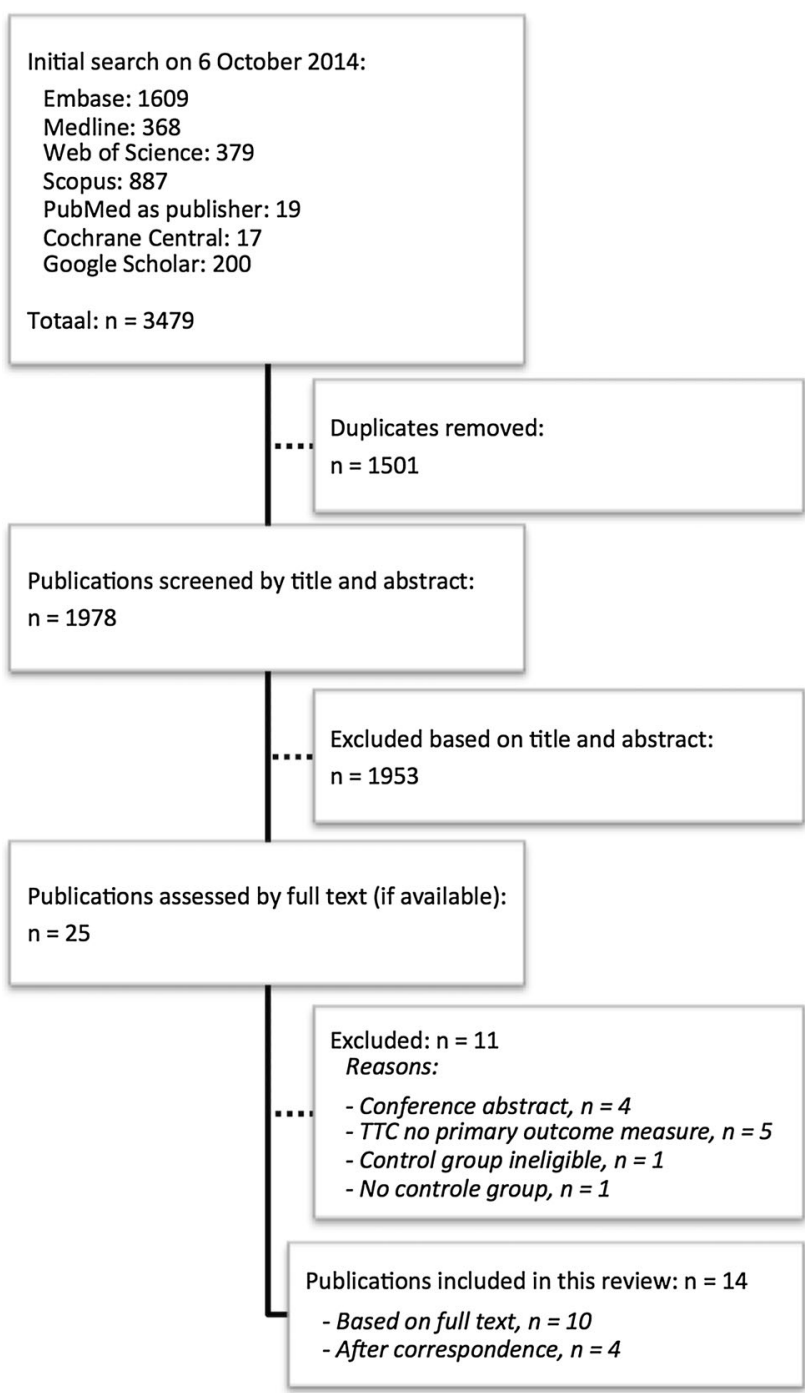

Fig. 1 Flowchart of the study selection procedure

[7, 16-28]. The initial consensus between the reviewers after screening of title and abstract was $99.4 \%$. Screening of the reference lists of the included papers did not result in the inclusion of additional studies. Four studies were included only after essential information was acquired through correspondence with the authors [19, 20, 25, 27]. Extra information was received for three other studies as well $[7,21,23]$.

\section{Study characteristics and quality}

The study characteristics are shown in Table 1. As expected, no randomized controlled trials were found on IBR and adjuvant chemotherapy. All included studies were retrospective cohort studies, of which one used matching to define a control cohort. It should be noted that Alderman et al. [16] and Vandergrift et al. [27] partly cover the same patient population for the entire year of 2003.

The results of the quality assessment are shown in Table 2. Few studies reported information about follow-up. In most studies patients were included if they received chemotherapy and therefore patients who started chemotherapy late may have been missed if follow-up was insufficient to identify them.

Table 3 shows the patient characteristics of the studies included in the current review. Few studies reported data on patient age, comorbidity, and risk factors for postoperative complications and even fewer studies reported these characteristics specifically for patients who had received chemotherapy. Therefore, it was not possible to provide an overview of these variables.

\section{Study heterogeneity}

The patient populations were compared regarding the inclusion criteria used and the available patient characteristics in order to determine the clinical heterogeneity. Due to lack of pertinent information, the legitimacy to do a meta-analysis was doubted. Moreover, an $\mathrm{I}^{2}$ of $98 \%$ was observed, after pooling the studies that used the same definition for time to chemotherapy and reported mean values and standard deviations [7, 17, 20, 22, 23], indicating a high statistical heterogeneity. We found no obvious explanation for this high statistical heterogeneity. Consequently, no meta-analysis was performed.

\section{Time to chemotherapy}

The included studies reported TTC in different formats (Table 4 and Fig. 2). Originally, seven studies reported TTC as a mean value and seven as a median value. The authors of one of the studies that reported medians provided us with means and standard deviations on request [7]. If the required information was available, the $95 \%$ confidence interval was computed for the mean differences. Alderman et al. [16] only reported values for two separate age groups instead of the total patient group.

In the twelve studies that measured TTC from surgery, it varied between 29 and 61 days in the IBR groups and between 21 and 60 days in the patient groups that received mastectomy only. Differences in TTC between the IBR groups and the mastectomy only groups varied widely from a 12.6 days reduction in average TTC after IBR to a delay of 11.9 days in average TTC after IBR [7, 16-20, 22-26, 28]. One study found a significantly shorter TTC after IBR [17], three studies found a statistically significant delay following IBR [7, 22, 24], six studies found no statistically significant difference in TTC between IBR and 
Table 1 Characteristics of the included studies on IBR and adjuvant chemotherapy

\begin{tabular}{|c|c|c|c|c|c|c|}
\hline & $\begin{array}{l}\text { Year of } \\
\text { publication }\end{array}$ & $\begin{array}{l}\text { Research } \\
\text { period }\end{array}$ & Country & Type $^{a}$ & Center & Patient recruitment (and extra data source) \\
\hline Alderman [16] & 2010 & 1997-2003 & USA & RCS & Multi & Prospectively maintained database \\
\hline Allweis [17] & 2002 & 1996-2000 & USA & RCS & Single & Hospital tumor registry (and medical records) \\
\hline Chang [18] & 2013 & $2003-2009$ & Australia & RCS & Single & Prospectively maintained database \\
\hline Eriksen $^{\mathrm{b}}[19]$ & 2011 & 1990-2004 & Sweden & RMCS & Single & Prospectively maintained database \\
\hline Hamahata $^{\mathrm{b}}[20]$ & 2013 & 2006-2011 & Japan & RCS & Single & Medical records \\
\hline $\mathrm{Kahn}^{\mathrm{b}}[21]$ & 2013 & $2008-2011$ & UK & RCS & Single & Prospectively maintained database (and medical records) \\
\hline Lee [22] & 2011 & $2008-2010$ & Korea & RCS & Single & Institutional electronic patient database and medical records \\
\hline Mortenson $^{\mathrm{b}}[23]$ & 2004 & 1995-2002 & USA & RCS & Single & Medical records \\
\hline Newman [24] & 1999 & $1990-1993$ & USA & RCS & Single & Prospectively maintained database \\
\hline $\operatorname{Rey}^{\mathrm{b}}[25]$ & 2005 & 1999-2002 & Italy & RCS & Single & $?$ \\
\hline Taylor [26] & 2004 & 1999-2002 & UK & RCS & Single & Regional tumor registry \\
\hline Vandergrift $^{\mathrm{b}}[27]$ & 2013 & 2003-2009 & USA & RCS & Multi & Prospectively maintained database (and medical records) \\
\hline Wilson [28] & 2004 & $1995-2000$ & UK & RCS & Single & $\begin{array}{l}\text { Database (and the case notes crosschecked with the } \\
\text { pharmacy records) }\end{array}$ \\
\hline Zhong $^{\mathrm{b}}[7]$ & 2012 & $2007-2010$ & Canada & RCS & Single & Prospectively maintained database \\
\hline
\end{tabular}

mastectomy only [18-20, 23, 25, 28], and two studies did not perform a statistical test for the comparison [16, 26].

Two studies used a different definition for TTC. Kahn et al. [21] measured TTC starting from the multidisciplinary decision to administer adjuvant treatment and reported a TTC of 31 days for IBR and of 29 days for mastectomy only, resulting in a statistically non-significant difference of 2 days. TTC was measured from pathological diagnosis by Vandergrift et al. [27], reporting 96.6 days for IBR and 79.8 days for mastectomy only, with a difference of 16.8 days. It was clarified via correspondence that this multidisciplinary decision was made after surgery (L. Romics Jr., personal communication), whereas the pathological diagnosis was made before the operation. These differences in definitions should be kept in mind when evaluating and comparing TTC published in the different studies.

Comparing the upper ranges gives insight of the TTC for the extremes in each cohort. Those maximum values were similar or lower for the IBR groups than for the mastectomy only groups. Furthermore, in 4 out of 6 studies presenting ranges, all patients started chemotherapy within 13 weeks after IBR.

Two studies performed a multivariate analysis correcting for various sociodemographic, clinical, therapeutic, and diagnostic factors. Alderman et al. [16] found a significantly shorter TTC for patients younger than 40 years after mastectomy only; at older ages no significant differences were found between IBR and mastectomy only. Vandergrift et al. [27] found a significantly shorter TTC following mastectomy only, even after multivariate correction.

\section{Other chemotherapy-related outcomes}

Six studies reported the number of patients that had received chemotherapy after a certain point in time in the comparison of IBR with mastectomy only. Different cutoff points were chosen: two studies chose 8 weeks, three studies chose 12 weeks, and one study reported the number of patients per 10 days. No statistically significant differences for this comparison were found for these cut-off points $[7,16,18,20,22,26]$. In patients with IBR, the few delays beyond 12 weeks after surgery were not related to the type of surgery, but due to social reasons and delayed diagnostic test results [7, 20].

Out of eight included studies which reported the occurrence of complications, two studies found significantly more complications in the IBR group [22, 23] and six studies did not find a significant difference (in one study after adjusting for confounders) between IBR and mastectomy only [7, 18-20, 24, 25]. Three studies evaluated 
Table 2 Quality assessment of the included studies on IBR and adjuvant chemotherapy

\begin{tabular}{|c|c|c|c|c|c|}
\hline Study & $\begin{array}{l}\text { Bias due to a non- } \\
\text { representative or ill- } \\
\text { defined sample of } \\
\text { patients }\end{array}$ & $\begin{array}{l}\text { Bias due to insufficiently long, or } \\
\text { incomplete follow-up, or } \\
\text { differences in follow-up between } \\
\text { treatment group }\end{array}$ & $\begin{array}{l}\text { Bias due to ill- } \\
\text { defined or } \\
\text { inadequately } \\
\text { measured } \\
\text { outcomes }\end{array}$ & $\begin{array}{l}\text { Bias due to inadequate } \\
\text { adjustment for all } \\
\text { important prognostic } \\
\text { factors }\end{array}$ & $\begin{array}{l}\text { Unclear or } \\
\text { inconsistent } \\
\text { reported } \\
\text { outcome } \\
\text { measure }\end{array}$ \\
\hline Alderman [16] & UL & UL & UL & $L^{f}$ & No \\
\hline Allweis [17] & UL & $?$ & UL & $L^{g}$ & $\operatorname{Yes}^{\mathrm{i}}$ \\
\hline Chang [18] & $\mathrm{UL}$ & $?$ & UL & $L^{g}$ & No \\
\hline Eriksen $^{a}[19]$ & UL & $\mathrm{L}^{\mathrm{c}}$ & UL & $L^{h}$ & No \\
\hline Hamahata $^{\mathrm{a}}[20]$ & UL & $?$ & UL & $L^{g}$ & Yes $^{\mathrm{i}}$ \\
\hline $\operatorname{Kahn}^{\mathrm{a}}[21]$ & UL & $?$ & $L^{d}$ & $L^{h}$ & No \\
\hline Lee [22] & $\mathrm{UL}$ & $?$ & UL & $L^{g}$ & No \\
\hline Mortenson $^{\mathrm{a}}$ [23] & UL & $?$ & UL & $L^{h}$ & Yes $^{\mathrm{i}}$ \\
\hline Newman [24] & UL & UL & UL & $L^{h}$ & No \\
\hline Rey $^{\mathrm{a}}[25]$ & $?^{\mathrm{b}}$ & UL & UL & $L^{g}$ & $\mathrm{Yes}^{\mathrm{j}}$ \\
\hline Taylor [26] & $\mathrm{UL}$ & $?$ & UL & $L^{h}$ & No \\
\hline Vandergrift $^{\mathrm{a}}[27]$ & UL & UL & $\mathrm{UL}^{\mathrm{e}}$ & $L^{f}$ & No \\
\hline Wilson [28] & $?^{\mathrm{b}}$ & $?$ & UL & $L^{h}$ & Yes $^{\mathrm{i}}$ \\
\hline Zhong $^{\mathrm{a}}$ [7] & UL & $?$ & UL & $L^{g}$ & No \\
\hline
\end{tabular}

$U L$ unlikely; ? unclear; L likely; IBR immediate breast reconstruction; CTx adjuvant chemotherapy; TTC Time to adjuvant chemotherapy

${ }^{a}$ Additional information about this paper was required through correspondence with the authors

b Patient selection unclear

${ }^{c}$ Lost to follow-up for TTC: 15 and $24 \%$ for IBR and mastectomy, respectively

d TTC measured from multidisciplinary decision to administer adjuvant treatments instead of final operation, allowing for other factors than type of operation to affect TTC, which is inconsistent with the study purpose

e Alternative definition for TTC, but consistent with the study purpose

${ }^{\mathrm{f}}$ Corrected for some but not all. For example type of reconstruction and smoking behavior were omitted

g Some data on possible confounders reported, but adjusted for none

${ }^{\mathrm{h}}$ Did not report data on possible confounders for patients receiving CTx

${ }^{\mathrm{i}}$ Different values for the same outcome measure reported

j Type of point estimator not stated (clarified by e-mail)

the effect of these complications on TTC. One study showed a significantly longer TTC for patients with complications compared to patients without complications [22]. However, for patients with complications none of these studies showed a statistically significant difference in TTC between the IBR and mastectomy only groups [20, 22, 23].

Four studies that looked at TTC after various types of breast reconstruction could not find statistically significant differences $[17,20,22,28]$. There were also no significant differences reported between IBR and mastectomy only in delay relative to planned initiation, dose reduction, delay during cycles or incomplete regimens [18, 19, 23, 26].

Besides TTC, omission of chemotherapy may influence oncological outcome. Only one study investigated this. Patients were included if they required adjuvant chemotherapy according to treatment guidelines. No statistically significant difference in omission of chemotherapy was found between the IBR and mastectomy group [16].

\section{Discussion}

This systematic review shows that IBR does not necessarily delay the start of adjuvant chemotherapy to a clinically relevant extent [9-13]. Differences in TTC between the IBR groups and the mastectomy only groups varied widely, ranging from a 12.6-day shorter TTC for IBR to a 16.6-day shorter TTC for mastectomy only. Out of 14 studies 10 studies reported a difference in TTC of less than a week 


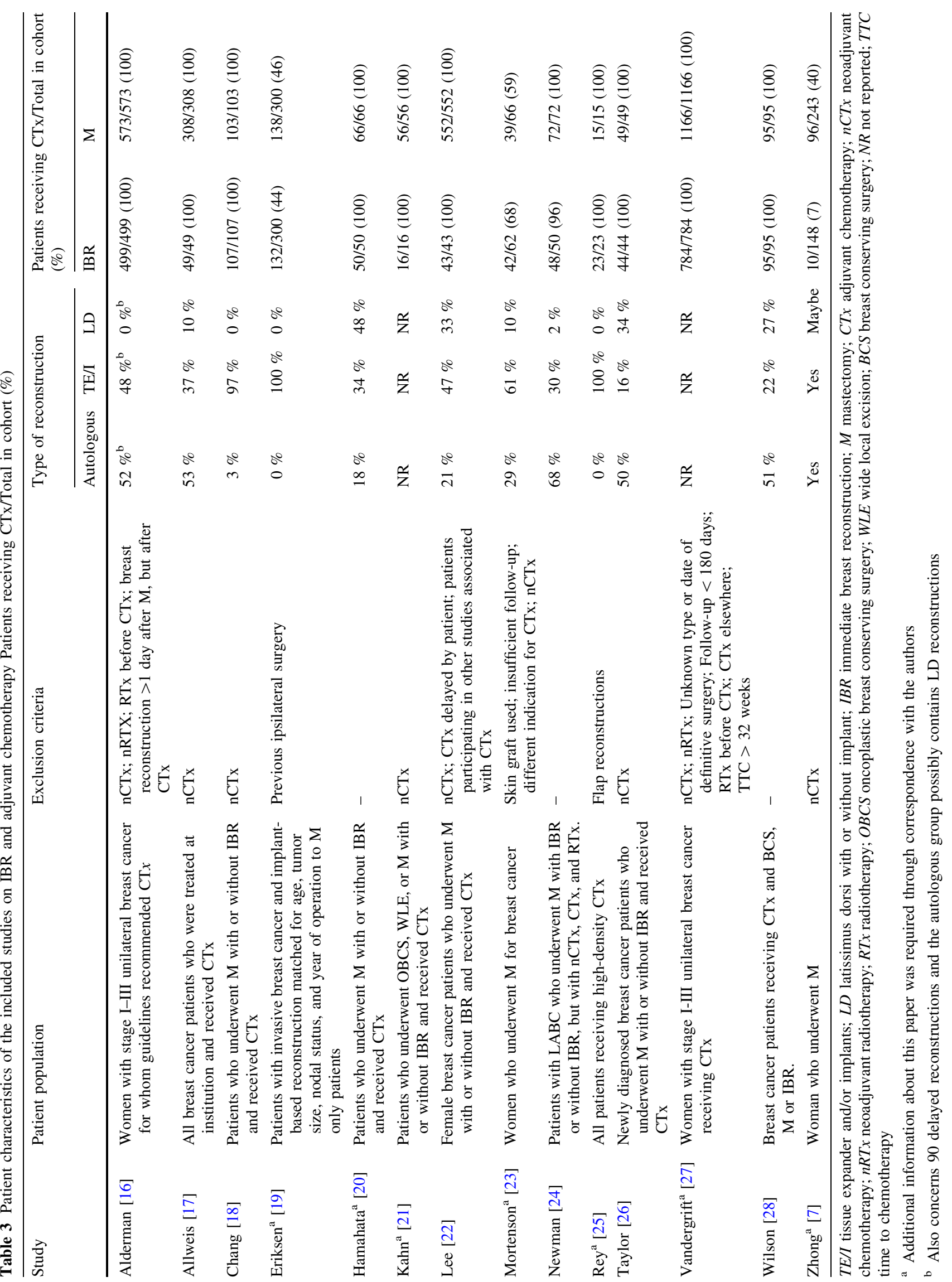




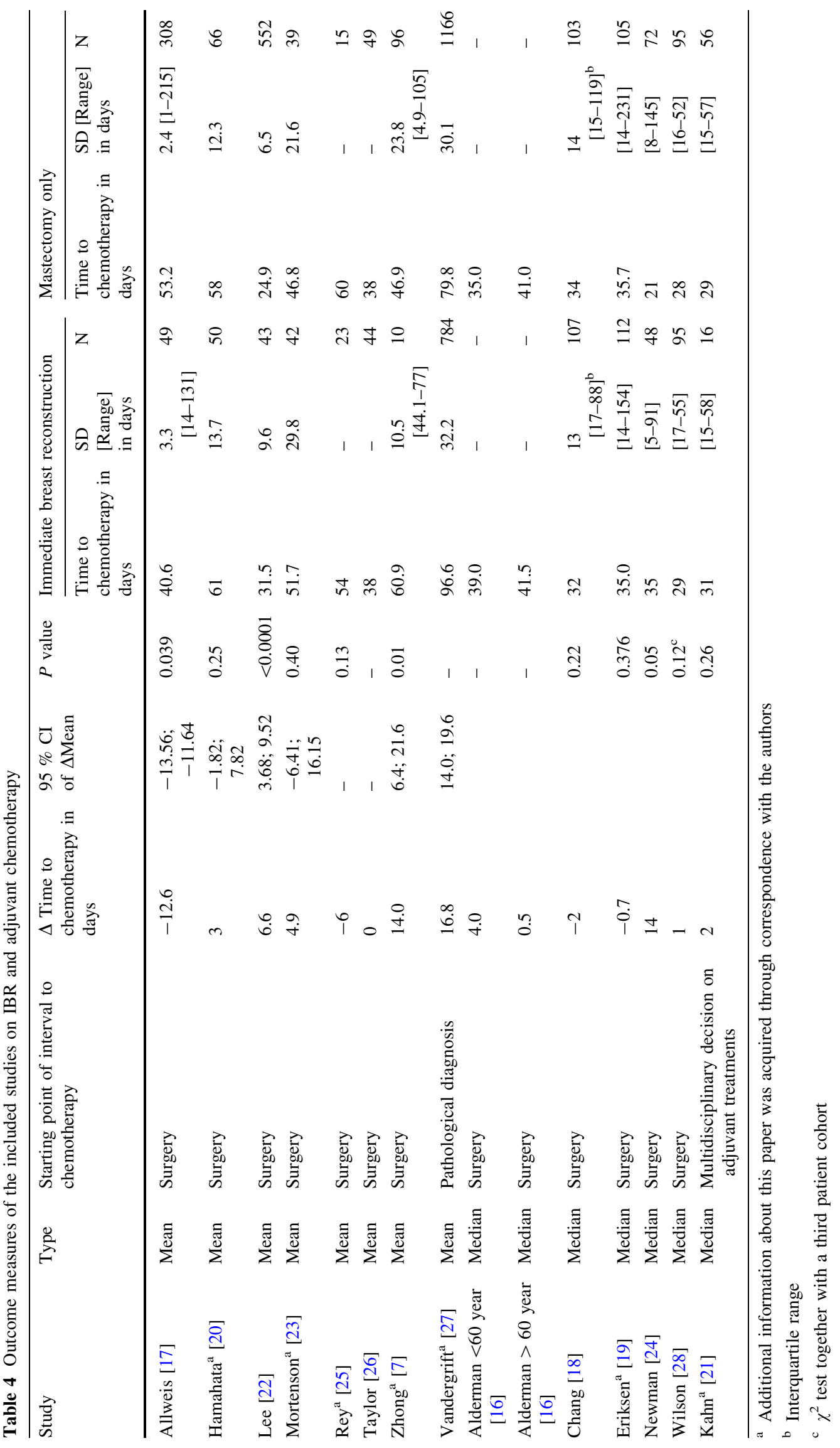




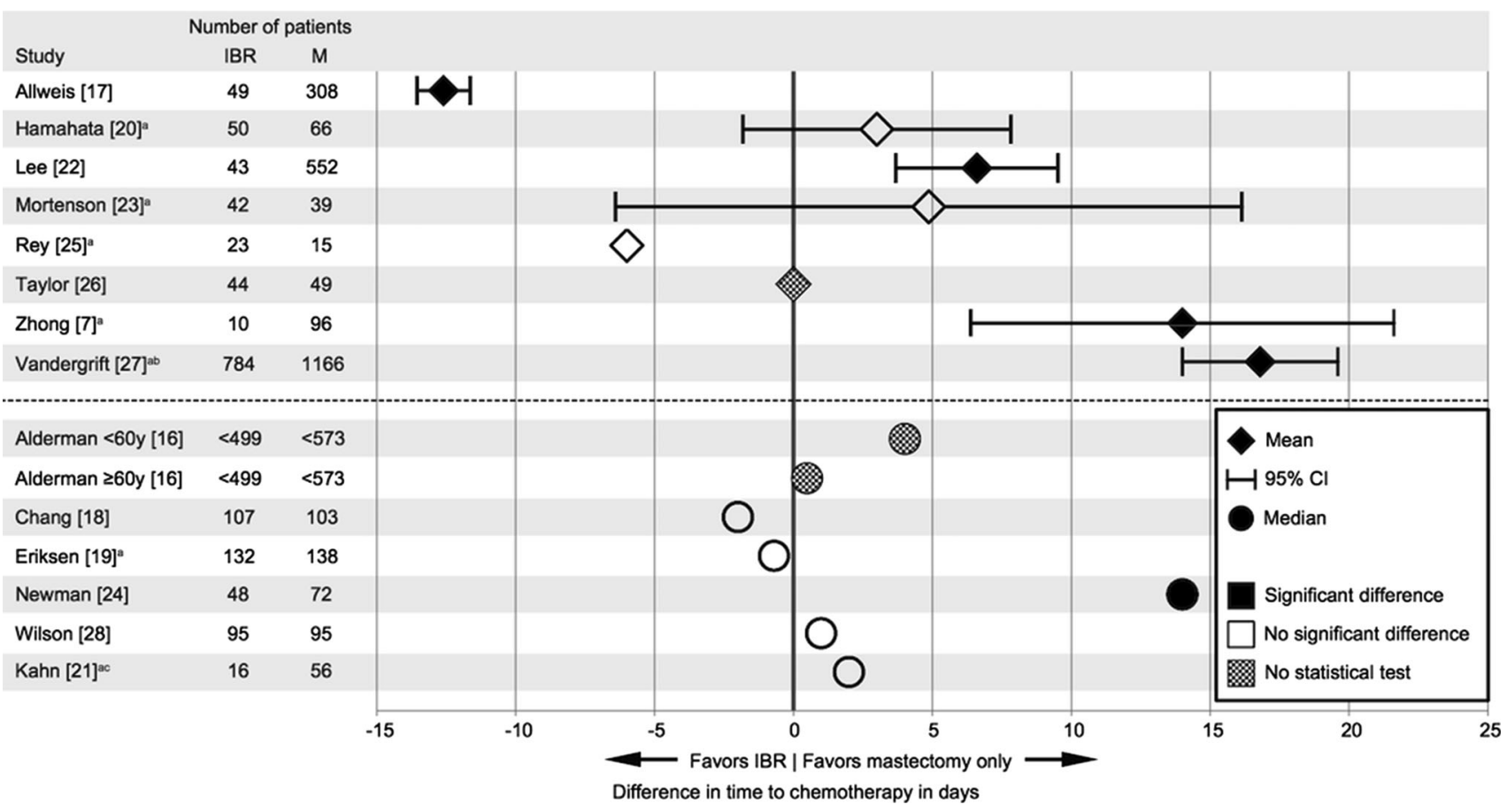

Fig. 2 Differences between IBR and mastectomy only in time to chemotherapy in days. $I B R$ immediate breast reconstruction; $M$ mas-

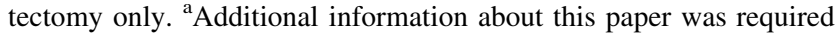

between these two groups. Two important reasons for delay of chemotherapy may equally apply to any surgery: difficulties in planning the surgery and surgical complications.

First, difficulties in planning a multidisciplinary surgery such as IBR, probably delays start of chemotherapy. This is an explanation for the average delay of 16.6 days found by Vandergrift et al. as they measured the interval from pathological diagnosis to chemotherapy [27]. In this approach factors other than the surgical procedure itself will affect the measurement. For example, planning IBR surgery most likely takes more time than planning a mastectomy only, due to additional outpatient visits of different specialists before surgery, more operation time, and extra surgeons are necessary for performing IBR compared to mastectomy. All other studies measured TTC as of the date of surgery or multidisciplinary decision and were therefore incapable of identifying possible delays due to logistical difficulties in planning IBR. Besides planning of the surgery, other factors may influence TTC, such as different local protocols and the person deciding on the actual start of chemotherapy. Delays due to difficulties planning surgery may be reduced in case adequate logistical measures are taken. For example, scheduling the availability of a combined oncologic and plastic surgery operation room for IBR at regular intervals. In case of relatively long absolute TTC, local treatment protocols should be reassessed. With the growing trend towards multidisciplinary approach in through correspondence with the authors. ${ }^{\mathrm{b}}$ Time to chemotherapy measured from pathological diagnosis. ${ }^{\mathrm{c}}$ Time to chemotherapy measured from multidisciplinary decision

health care generally, efficient planning and adequate protocols are key to avoid unnecessary delays. Such difficulties should not reduce the usage of multidisciplinary therapies such as IBR.

Second, the studies included in this review suggest that complications after IBR do not result in longer TTC compared to complications after mastectomy only. Complications in general are considered to delay chemotherapy and this was confirmed in one study recording a longer TTC for patients with complications compared to patients without complications [22]. There was no conclusive evidence that IBR gave rise to more complications than mastectomy only. Six studies found no difference in complication rate $[7,18-20,24,25]$. One of them reported more complications with IBR in unadjusted data, but not after correction for confounders (previous surgery, previous radiotherapy, bilateral surgery) [7]. Two studies found more complications in the IBR group, but did not collect data on these confounders or did not correct for them while there were clear differences between the treatment groups $[22,23]$. One study showed bilateral breast surgery results in more complications, although not proportionally more than two unilateral breast surgeries. Since the feasibility of IBR might increase the demand on contralateral prophylactic mastectomy by women with unilateral breast cancer, as a consequence the absolute number of complications may increase compared to unilateral breast surgery [29]. 
None of these studies showed a statistically significant difference in TTC between the IBR and mastectomy only groups for patients with complications [20, 22, 23].

The clinical relevance of a delay must be assessed in relation to absolute findings of TTC. No study reported an average interval from surgery to start of chemotherapy longer than the clinically relevant limit of 12 weeks after surgery [12]. As mentioned before, variation in commencement date of chemotherapy within 3 months maximum after surgery does not seem to have a significant effect on survival rates [9-13]. Consequently, no major impact on oncological safety is to be expected due to the reported average delays in TTC.

There are a few limitations to this review. Our quality assessment showed that most studies were potentially subject to bias. However, this was not to such an extent that a specific study had to be excluded from the review. It proved difficult to specifically examine the effect of IBR on TTC, because the majority of the studies reported general patient characteristics only without possible confounders or correcting for them. Some studies showed considerable differences in patient characteristics between the IBR and mastectomy only group. Furthermore, it is plausible that different types of breast reconstruction may have different effects on TTC, but only four studies investigated this issue. These studies did not find statistically significant differences, which may be due to small patient numbers $[17,20,22,28]$. In two studies that corrected for various possible confounders, a statistically significant delay in TTC associated with IBR was found [16, 27]. In one study this finding was restricted to patients under 40 years of age [16]. Many studies used the administration of adjuvant chemotherapy as an inclusion criterion. It is possible that certain patients were missed out, in which case they received chemotherapy at another institution or at a later point in time than the follow-up period. Unfortunately, follow-up was not specifically reported in most studies. One study explicitly recorded that patients whose chemotherapy started more than 32 weeks after diagnosis were excluded [27]. As a result there may be bias, because patients with chemotherapy delayed beyond that period are taken out of the equation.

Since most studies report only on TTC as an average, they do not provide a basis for identifying outliers. Increased oncological risk may impact the few patients subject to the longest delays. Regardless, there was no significant difference in the number of patients with a TTC longer than 8 between IBR and mastectomy only [16, 18]. The same goes for patients with a TTC longer than 12 weeks [7, 20, 22]. The reasons given for the limited number of delays beyond 12 weeks after surgery were unrelated to the type of surgery, but due to social reasons and delayed diagnostic test results. The upper ranges of TTC were favorable for IBR.

Patients most susceptible for negative effects of delay may be those with the most aggressive tumors and metastatic disease. However, it is not really clear what the effect of delay in TTC is for those patients. Consequently, any delay due to IBR is of greater concern in this particular patient population. This systematic review did not include an analysis of the effect of IBR on TTC for specific subgroups potentially at risk [10], because the included studies did not provide data for such subgroups.

Beside the effect of IBR on TTC, there are concerns about postoperative morbidity resulting from the combination of IBR with radiotherapy or chemotherapy. First, a meta-analysis showed a negative effect on postoperative morbidity in patients receiving immediate breast reconstruction in case of adjuvant radiotherapy. However, delaying breast reconstruction until radiotherapy is finished did not improve postoperative morbidity. Autologous reconstruction resulted in less postoperative morbidity than implant-based reconstructions [30]. Second, IBR seems safe in patients who received neoadjuvant chemotherapy, as this did not increase the complication rate [31]. The marginal delay in delivery of adjuvant chemotherapy could be an argument to support the growing popularity of neoadjuvant chemotherapy. Third, one review suggested that the combination of IBR and adjuvant chemotherapy does not have a negative effect on surgical complications, postoperative wound healing, chance of reconstructive failure, or esthetic outcomes [32]. Finally, the effect of IBR on the oncological outcome in terms of recurrences is an issue. However, a meta-analysis comparing local and systemic recurrence rates after IBR and mastectomy only in locally advanced breast cancer patients did not show statistically significant differences [33].

Presently available evidence shows that IBR is safe with regard to the timing of adjuvant chemotherapy. Nevertheless, future developments in breast cancer treatment may require a reassessment. Future studies should identify and report possible confounders and adjust for them. In addition, inclusion and analysis of local protocols on the planning of adjuvant chemotherapy will be helpful to put the findings into perspective. Most studies included in this review had risk of bias, which further research should try to avoid, in order to allow for more reliable conclusions. It would seem important to further analyze the effect of IBR on other chemotherapy-related outcomes, such as dose reduction, delay during cycles, incomplete regimens or omission, as these also represent aspects of oncological safety. Finally, a systematic evaluation of the effect of adjuvant chemotherapy on breast reconstruction in terms of complication rates, esthetic outcome, and patient 
satisfaction would be required for more detailed and conclusive findings outside the scope of this review.

In conclusion, after critical appraisal of the current literature, we found that IBR does not necessarily delay chemotherapy to a clinically relevant extent. With efficient logistics and adequate treatment protocols the risk of crossing the described 12-week barrier can be avoided. This would suggest that in general IBR is a valid option for non-metastatic breast cancer patients.

Acknowledgments The authors would like to thank Ms. B.S. NiëlWeise, M.D., epidemiologist, from the Kennisinstituut van Medisch Specialisten for her assistance with the study design and statistical analysis and Mr W.M. Bramer, biomedical information specialist, for help with the literature search.

\section{Compliance with ethical standards}

Conflict of interests The authors declare that they have no conflict of interest.

Open Access This article is distributed under the terms of the Creative Commons Attribution-NonCommercial 4.0 International License (http://creativecommons.org/licenses/by-nc/4.0/), which permits any noncommercial use, distribution, and reproduction in any medium, provided you give appropriate credit to the original author(s) and the source, provide a link to the Creative Commons license, and indicate if changes were made.

\section{References}

1. American Cancer Society (2013) Breast cancer facts \& figures 2013-2014. American Cancer Society, Atlanta

2. International Agency for Research on Cancer (2008) World cancer report 2008. Lyon, France

3. World Health Organization (2014) Global health estimates 2014 summary tables: deaths by cause, age and sex, 2000-2012. Global Health Estimates, Geneva

4. Berry MG, Gomez KF (2013) Surgical techniques in breast cancer: an overview. Surgery (Oxford) 31(1):32-36. doi:10.1016/ j.mpsur.2012.10.013

5. Dragun AE, Huang B, Tucker TC, Spanos WJ (2012) Increasing mastectomy rates among all age groups for early stage breast cancer: a 10-year study of surgical choice. Breast J 18(4):318-325. doi:10.1111/j.1524-4741.2012.01245.x

6. Gopie JP, Hilhorst MT, Kleijne A, Timman R, Menke-Pluymers MB, Hofer SO et al (2011) Women's motives to opt for either implant or DIEP-flap breast reconstruction. J Plast Reconstr Aesthet Surg 64(8):1062-1067. doi:10.1016/j.bjps.2011.03.030

7. Zhong T, Hofer SOP, McCready DR, Jacks LM, Cook FE, Baxter N (2012) A comparison of surgical complications between immediate breast reconstruction and mastectomy: the impact on delivery of chemotherapy-an analysis of 391 procedures. Ann Surg Oncol 19:560-566

8. Wanzel KR, Brown MH, Anastakis DJ, Regehr G (2002) Reconstructive breast surgery: referring physician knowledge and learning needs. Plast Reconstr Surg 110(6):1441-1450. doi:10. 1097/01.PRS.0000030458.86726.50

9. Cold S, During M, Ewertz M, Knoop A, Moller S (2005) Does timing of adjuvant chemotherapy influence the prognosis after early breast cancer? Results of the Danish Breast Cancer Cooperative Group (DBCG). Br J Cancer 93(6):627-632. doi:10.1038/ sj.bjc. 6602734

10. Colleoni M, Bonetti M, Coates AS, Castiglione-Gertsch M, Gelber RD, Price K et al (2000) Early start of adjuvant chemotherapy may improve treatment outcome for premenopausal breast cancer patients with tumors not expressing estrogen receptors. The International Breast Cancer Study Group. J Clin Oncol 18(3):584-690

11. Jara Sanchez C, Ruiz A, Martin M, Anton A, Munarriz B, Plazaola A et al (2007) Influence of timing of initiation of adjuvant chemotherapy over survival in breast cancer: a negative outcome study by the Spanish Breast Cancer Research Group (GEICAM). Breast Cancer Res Treat 101(2):215-223. doi:10. 1007/s10549-006-9282-0

12. Lohrisch C, Paltiel C, Gelmon K, Speers C, Taylor S, Barnett J et al (2006) Impact on survival of time from definitive surgery to initiation of adjuvant chemotherapy for early-stage breast cancer. J Clin Oncol 24(30):4888-4894. doi:10.1200/JCO.2005.01.6089

13. Shannon C, Ashley S, Smith IE (2003) Does timing of adjuvant chemotherapy for early breast cancer influence survival? J Clin Oncol 21(20):3792-3797. doi:10.1200/JCO.2003.01.073

14. Hayden JA, van der Windt DA, Cartwright JL, Cote P, Bombardier C (2013) Assessing bias in studies of prognostic factors. Ann Intern Med 158(4):280-286. doi:10.7326/0003-4819-158-4201302190-00009

15. Hatala R, Keitz S, Wyer P, Guyatt G, Evidence-Based Medicine Teaching Tips Working G (2005) Tips for learners of evidencebased medicine: 4 . Assessing heterogeneity of primary studies in systematic reviews and whether to combine their results. CMAJ 172 (5):661-665. doi: 10.1503/cmaj.1031920

16. Alderman AK, Collins ED, Schott A, Hughes ME, Ottesen RA, Theriault RL et al (2010) The impact of breast reconstruction on the delivery of chemotherapy. Cancer 116:1791-1800

17. Allweis TM, Boisvert ME, Otero SE, Perry DJ, Dubin NH, Priebat DA (2002) Immediate reconstruction after mastectomy for breast cancer does not prolong the time to starting adjuvant chemotherapy. Am J Surg 183:218-221

18. Chang RJC, Kirkpatrick K, De Boer RH, Bruce Mann G (2013) Does immediate breast reconstruction compromise the delivery of adjuvant chemotherapy? Breast 22:64-69

19. Eriksen C, Frisell J, Wickman M, Lidbrink E, Krawiec K, Sandelin K (2011) Immediate reconstruction with implants in women with invasive breast cancer does not affect oncological safety in a matched cohort study. Breast Cancer Res Treat 127:439-446

20. Hamahata A, Kubo K, Takei H, Saitou T, Hayashi Y, Matsumoto $H$ et al (2013) Impact of immediate breast reconstruction on postoperative adjuvant chemotherapy: a single center study. Breast Cancer 22:1-5

21. Kahn J, Barrett S, Forte C, Stallard S, Weiler-Mithoff E, Doughty JC et al (2013) Oncoplastic breast conservation does not lead to a delay in the commencement of adjuvant chemotherapy in breast cancer patients. Eur J Surg Oncol 39(8):887-891

22. Lee J, Lee SK, Kim S, Koo MY, Choi MY, Bae SY et al (2011) Does immediate breast reconstruction after mastectomy affect the initiation of adjuvant chemotherapy? J Breast Cancer 14:322-327

23. Mortenson MM, Schneider PD, Khatri VP, Stevenson TR, Whetzel TP, Sommerhaug EJ et al (2004) Immediate breast reconstruction after mastectomy increases wound complications: however, initiation of adjuvant chemotherapy is not delayed. Arch Surg 139:988-991

24. Newman LA, Kuerer HM, Hunt KK, Ames FC, Ross MI, Theriault R et al (1999) Feasibility of immediate breast reconstruction for locally advanced breast cancer. Ann Surg Oncol 6:671-675 
25. Rey P, Martinelli G, Petit JY, Youssef O, De Lorenzi F, Rietjens $M$ et al (2005) Immediate breast reconstruction and high-dose chemotherapy. Ann Plast Surg 55:250-254

26. Taylor CW, Kumar S (2005) The effect of immediate breast reconstruction on adjuvant chemotherapy. Breast 14:18-21

27. Vandergrift JL, Niland JC, Theriault RL, Edge SB, Wong YN, Loftus LS et al (2013) Time to adjuvant chemotherapy for breast cancer in National Comprehensive Cancer Network institutions. J Natl Cancer Inst 105:104-112

28. Wilson CR, Brown IM, Weiller-Mithoff E, George WD, Doughty JC (2004) Immediate breast reconstruction does not lead to a delay in the delivery of adjuvant chemotherapy. Eur J Surg Oncol 30:624-627

29. Miller ME, Czechura T, Martz B, Hall ME, Pesce C, Jaskowiak N et al (2013) Operative risks associated with contralateral prophylactic mastectomy: a single institution experience. Ann Surg Oncol 20(13):4113-4120. doi:10.1245/s10434-013-3108-1
30. Barry M, Kell MR (2011) Radiotherapy and breast reconstruction: a meta-analysis. Breast Cancer Res Treat 127(1):15-22. doi:10.1007/s10549-011-1401-x

31. Song J, Zhang X, Liu Q, Peng J, Liang X, Shen Y et al (2014) Impact of neoadjuvant chemotherapy on immediate breast reconstruction: a meta-analysis. PLoS ONE 9(5):e98225. doi:10. 1371/journal.pone.0098225

32. Oh E, Chim H, Soltanian HT (2012) The effects of neoadjuvant and adjuvant chemotherapy on the surgical outcomes of breast reconstruction. J Plast Reconstr Aesthet Surg 65(10):e267-280. doi:10.1016/j.bjps.2012.04.053

33. Gieni M, Avram R, Dickson L, Farrokhyar F, Lovrics P, Faidi S et al (2012) Local breast cancer recurrence after mastectomy and immediate breast reconstruction for invasive cancer: a metaanalysis. Breast 21(3):230-236. doi:10.1016/j.breast.2011.12.013 